\title{
Aortic size, distensibility, and pulse wave velocity changes with aging: longitudinal analysis from Multi-Ethnic Study of Atherosclerosis (MESA)
}

\author{
Chia-Ying Liu", Doris Chen ${ }^{1}$, Gisela Teixido-Tura², Atul R Chugh ${ }^{1}$, Alban Redheuil ${ }^{3}$, Antoinette S Gomes ${ }^{4}$, \\ Martin R Prince ${ }^{5}$, William Hundley ${ }^{6}$, David A Bluemke” ${ }^{7}$ Joao A Lima ${ }^{1}$
}

From 15th Annual SCMR Scientific Sessions

Orlando, FL, USA. 2-5 February 2012

\section{Summary}

Arterial stiffening is related to an intricate interplay between aging and other cardiovascular risk factors. The aortic arch accounts for most of the vascular buffering function and is primarily involved in arterial stiffening. MRI has been used to noninvasively measure strain, distensibility, and pulse wave velocity of the ascending aorta. We report aortic size and stiffness changes over mid to late adulthood in longitudinal comparisons with MRI over a 10-year period in the MESA cohort.

\section{Background}

Changes in the human cardiovascular system are an important aspect of aging. Arterial stiffness, a major determinant of increased systolic pulse pressure, is associated with aging and with the incidence of stroke, ischemic heart disease and heart failure. The proximal aorta accounts for most of the global arterial stiffening and subsequent complications. Aortic stiffness can be described by changes in aortic dimensions such as strain (proportional change of aortic area to the minimum area), as well as, combined with pressure, distensibility (relative dimensional change related to changes in local pressure). Another approach to aortic stiffness is the measurement of pulse wave velocity (PWV). While these changes with advancing age have been established in cross-sectional studies, few longitudinal data exist on the evolution of aortic biomechanical parameters. The aims of this study are to assess the longitudinal changes in central aortic size and stiffness measured with MRI over a 10 -year period in the MESA cohort.

\section{Methods}

226 longitudinal studies with aortic imaging by MRI were analyzed. MRI was performed at first in MESA1 (baseline, 2000-2001), and then in MESA5 (ten-year follow-up, 2010-2011) after ten years of follow-up. Both exams used 1.5-T whole-body MRI systems and phase contrast cine gradient echo sequence with ECG gating to evaluate aortic size and stiffness. Aortic sagittal oblique plane with black blood sequence was acquired to position the aortic imaging and allowed for the measurement of the distance between the ascending and descending aorta. Images of the ascending and descending aorta were obtained in the transverse plane at the level of the right pulmonary artery perpendicular to the vessel lumen. Aorta analysis was performed using a validated automated software (ARTFUN. INSERM U678). Aortic size, strain and distensibility were reported in the ascending aorta.

\section{Results}

Table 1 lists diastolic aortic size and stiffness aortic parameters measured in baseline and ten-year follow-up stratified by gender. Aortic area and PWV increased significantly with aging. Strain and distensibility of the ascending aorta were also reduced over time.

Table 2 displays the baseline values for aortic dimensions and aortic stiffness parameters and their absolute changes during follow-up. Ascending aortic area increased more markedly in men than in women during follow-up whereas stiffness parameters changes over time were similar between men and women.

1Johns Hopkins University, Baltimore, MD, USA

Full list of author information is available at the end of the article

(c) 2012 Liu et al; licensee BioMed Central Ltd. This is an open access article distributed under the terms of the Creative Commons Attribution License (http://creativecommons.org/licenses/by/2.0), which permits unrestricted use, distribution, and reproduction in any medium, provided the original work is properly cited. 
Table 1 Diastolic aortic size and stiffness aortic parameters measured in baseline and ten-year follow-up

\begin{tabular}{|c|c|c|c|}
\hline Women $(\mathrm{N}=131)$ & Baseline & Ten-year follow up & Paired test $p$-value \\
\hline Diastolic aortic size $(\mathrm{cm} 2)$ & $7.2 \pm 1.5$ & $8.1 \pm 1.7$ & $<0.001$ \\
\hline Ascending aorta Strain (\%) & $7.8 \pm 3.9$ & $7.2 \pm 3.7$ & 0.081 \\
\hline Ascending aorta Distensibility (mmhg-1) & $1.5 \pm 0.9$ & $1.4 \pm 0.9$ & .177 \\
\hline Aortic arch Pulse wave velocity (m/s) & $7.2 \pm 2.9$ & $8.3 \pm 4.1$ & $<0.001$ \\
\hline Men $(\mathrm{N}=95)$ & Baseline & Ten-year follow up & Paired test $\mathrm{p}$-value \\
\hline Diastolic aortic size $(\mathrm{cm} 2)$ & $8.6 \pm 1.7$ & $10.1 \pm 2.0$ & $<0.001$ \\
\hline Ascending aorta Strain (\%) & $7.4 \pm 4.1$ & $6.6 \pm 3.6$ & 0.086 \\
\hline Ascending aorta Distensibility (mmhg-1) & $1.6 \pm 1.0$ & $1.4 \pm 1.0$ & 0.191 \\
\hline Aortic arch Pulse wave velocity (m/s) & $7.2 \pm 3.0$ & $8.2 \pm 3.5$ & 0.011 \\
\hline GLOBAL (N=226) & Baseline & Ten-year follow up & Paired test $p$-value \\
\hline Diastolic aortic size (cm2) & $7.8 \pm 1.7$ & $8.9 \pm 2.1$ & $<0.001$ \\
\hline Ascending aorta Strain (\%) & $7.6 \pm 4.0$ & $7.0 \pm 3.6$ & 0.014 \\
\hline Ascending aorta Distensibility (mmhg-1) & $1.5 \pm 1.0$ & $1.4 \pm 0.9$ & 0.059 \\
\hline Aortic arch Pulse wave velocity $(\mathrm{m} / \mathrm{s})$ & $7.2 \pm 2.9$ & $8.3 \pm 3.9$ & $<0.001$ \\
\hline
\end{tabular}

Table 2 The baseline values for aortic dimensions and aortic stiffness parameters and their absolute changes during follow-up

\begin{tabular}{|c|c|c|c|c|}
\hline & GLOBAL (N=226) & Women $(\mathrm{N}=131)$ & Men $(\mathrm{N}=95)$ & $p$-value \\
\hline Age (years) at baseline & $59.4 \pm 8.4$ & $59.2 \pm 8.3$ & $59.7 \pm 8.6$ & 0.646 \\
\hline Diastolic aortic size $(\mathrm{cm} 2)$ at baseline & $7.8 \pm 1.7$ & $7.2 \pm 1.5$ & $8.6 \pm 1.7$ & $<0.001$ \\
\hline Weight at baseline $(\mathrm{kg})$ & $82.5 \pm 16.5$ & $78.9 \pm 16.9$ & $87.4 \pm 14.8$ & $<0.001$ \\
\hline Height at baseline (cm) & $168.4 \pm 10.1$ & $162.6 \pm 7.1$ & $176.5 \pm 7.9$ & $<0.001$ \\
\hline Ascending aorta Strain (\%) at baseline & $7.6 \pm 4.0$ & $7.8 \pm 3.9$ & $7.4 \pm 4.1$ & 0.370 \\
\hline Ascending aorta Distensibility (mmhg-1) at baseline & $1.5 \pm 1.0$ & $1.5 \pm 0.9$ & $1.6 \pm 1.0$ & 0.641 \\
\hline Aortic arch Pulse wave velocity $(\mathrm{m} / \mathrm{s})$ at baseline & $7.2 \pm 2.9$ & $7.2 \pm 2.9$ & $7.2 \pm 3.0$ & 0.892 \\
\hline Size change $(\mathrm{cm} 2)$ & $1.2 \pm 1.2$ & $0.9 \pm 1.1$ & $1.5 \pm 1.3$ & $<0.001$ \\
\hline Strain change $(\%)$ & $-0.7 \pm 4.1$ & $-0.6 \pm 3.9$ & $-0.8 \pm 4.4$ & 0.728 \\
\hline Distensibility change (mmhg-1) & $-0.15 \pm 1$ & $-0.1 \pm 1$ & $-0.2 \pm 1$ & 0.749 \\
\hline Pulse wave velocity change $(\mathrm{m} / \mathrm{s})$ & $1.0 \pm 3.4$ & $1.1 \pm 3.5$ & $0.9 \pm 3.2$ & 0.604 \\
\hline
\end{tabular}

\section{Conclusions}

We report aortic size and stiffness changes over mid to late adulthood in longitudinal comparisons. Further analyses will reveal the correlates of these alterations in multi-ethnic cohorts.

\section{Funding}

The study is supported by N01 HC 95168, Multi-Ethnic Study of Atherosclerosis (MESA), National Heart, Lung, and Blood Institute (NHLBI).
USA. ${ }^{7}$ Radiology and Imaging Sciences, National Institutes of Health, Bethesda, MD, USA.

Published: 1 February 2012

doi:10.1186/1532-429X-14-S1-P126

Cite this article as: Liu et al: Aortic size, distensibility, and pulse wave velocity changes with aging: longitudinal analysis from Multi-Ethnic Study of Atherosclerosis (MESA). Journal of Cardiovascular Magnetic Resonance 2012 14(Suppl 1):P126.

\footnotetext{
Author details

1Johns Hopkins University, Baltimore, MD, USA. ${ }^{2}$ Department of Cardiology, Hospital General Universitari Vall dHebron, Barcelona, Spain. ${ }^{3}$ European Hospital Georges Pompidou APHP, University of Paris Descartes and INSERM U678, Paris, France. ${ }^{4}$ Department of Radiology, University of California Los Angeles, Los Angeles, CA, USA. ${ }^{5}$ Department of Radiology, Weill Medical College of Cornell University, New York, NY, USA. 'Division of Cardiology, Deparment of Internal Medicine, Wake Forest University, Winston-Salem, NC,
} 